\title{
Secure and privacy-preserving RFID Authentication Scheme for Internet of Things Applications
}

\author{
Manik Lal Das ${ }^{1}$, Pardeep Kumar ${ }^{2}$, and Andrew Martin ${ }^{3}$ \\ ${ }^{1}$ DA-IICT, Gandhinagar, India. \\ ${ }^{2}$ Swansea University, United Kingdom. \\ ${ }^{3}$ University of Oxford, United Kingdom. *
}

\begin{abstract}
Privacy issue has become a crucial concern in Internet of Things (IoT) applications ranging from home appliances to vehicular networks. RFID system has found enormous scope in IoT applications such as consumer electronics, healthcare, tracking objects and transport system. In this paper, we present a privacy preserving authentication protocol for RFID system. The protocol provides mutual authentication of tag and reader, and preserves privacy of tags. We analyze the proposed protocol against active adversary and show that the protocol preserves narrow-strong privacy of the tags. We compare our protocol with the related work and show its usefulness in wide range of IoT applications.
\end{abstract}

Keywords: Internet of Things, RFID security, Privacy, Authentication, Elliptic Curves.

\section{Introduction}

With the advancement of Information and Communication technology Internet of Things (IoT) can smartly integrate several contemporary technologies (as shown in Fig. 1), which can leverage connecting any physical objects to cyberspace. Smart objects along with smart services will make cyberworld and physical world interacting intelligently anytime, anywhere and anything in our real lives. Some of the key challenges of IoT include a global standard, security and privacy, objects identification, trust, ownership, integration and regulation. Out of several contemporary technologies in IoT, Radio Frequency IDentification (RFID) system has found enormous applications in IoT applications, such as supply chain, access control, transport, healthcare, identity management, consumer electronics, home appliances and smart cities [1-4]. Furthermore, RFID chip-based object identification provides effective maintainability, flexibility, and automatic clearing and settlement.

An RFID system consists of a set of tags, readers, and a back-end server. A tag is

* Corresponding author: Manik Lal Das. Email: maniklal@gmail.com, Telephone: +9179-30510617, Fax: +91-79-30520010 
composed of a microchip and a transponder, whereas, a reader has one or more transceivers which emit radio waves by which passive tags respond back to the reader. The back-end server is assumed to be a trusted entity, connected to the reader securely, that keeps tags' and readers' information in its database. Nowadays, active tags, equipped with more computing resources in comparison to passive tags, can find applications like smart enthronement, where a tag-enabled object can talk wirelessly to the reader and then, the reader can send object's information to the back-end server.

Security and privacy are two equally important concerns in RFID system. On one hand, security of an RFID system primarily aims to identify legitimate tags. Although tags' authentication is the primary goal, reader's authentication is also important for some applications where a bogus reader could steal tags' data. On the other hand, privacy property ensures tag-enabled object not be traced by unauthorized party, because compromising the privacy of the tag-enabled object will reveal the identity information of the tag to unauthorized party, which in turn, leads to information leakage of the tag-enabled object. As a result, the goal of an RFID protocol should authenticate tags without revealing tags' identity to unauthorized party.

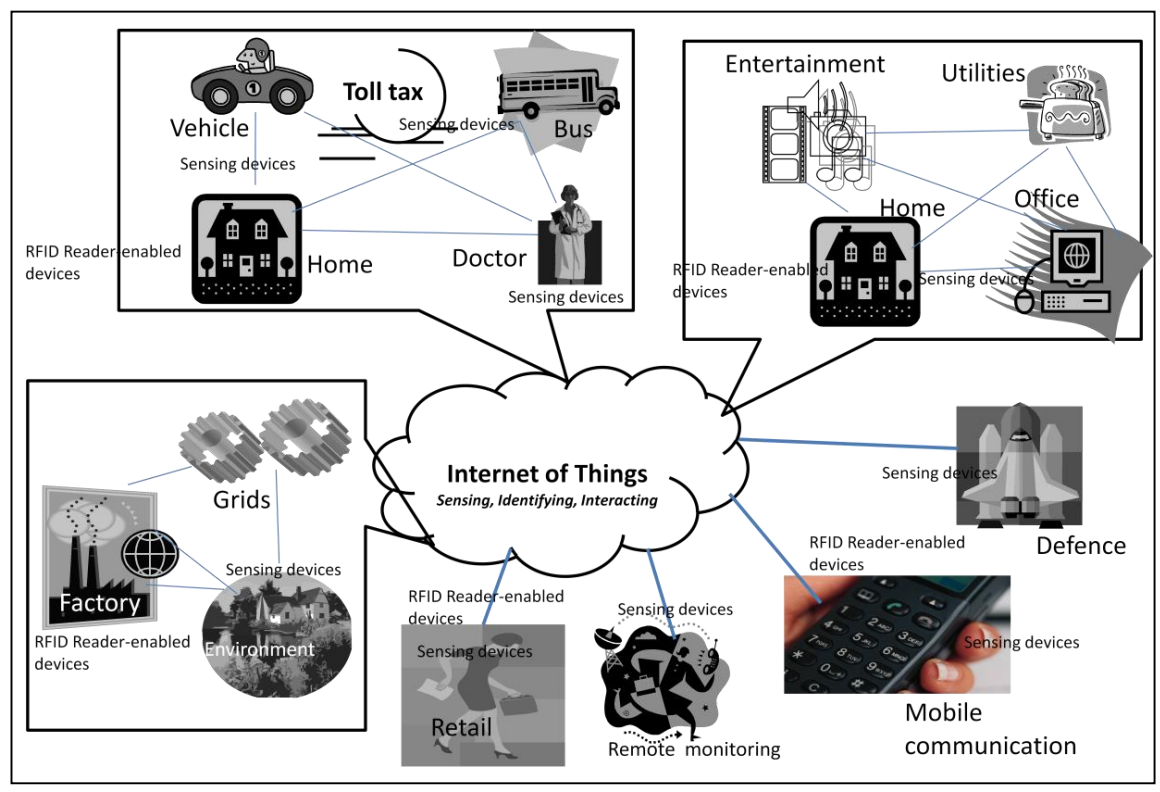

Fig. 1. IoT Scenarios and Applications [15] 
Related Work Recently, a large number of RFID authentication protocols have been proposed focusing on IoT. For instance, in [5], Fan et al. proposed a lightweight privacy-preserving RFID-based scheme with a focus on medical IoT. To ensure reliable and secure access to medical information of patients, an RFID tag sends patient's information to the reader over wireless communication. Under this scheme, a tag computes bit-wise XORing operations with a different value for each session and updates the secret key. The authors claimed that their scheme is secure against replay and denial of service attacks, and it can preserve the privacy of a tag. However, the scheme required high communicational cost and it may have (high) risk of the server impersonation attack. In another research, Rahman et al. proposed a privacy preserving framework focusing on RFID enabled healthcare in [6]. To maintain the scalability and privacy problems, the authors choose to implement a group based communications where each tag has at least one identifier common with at least two group members. However, in this scheme, the reader may incur high (computational and communicational) overhead if there are high numbers of tags in a group.

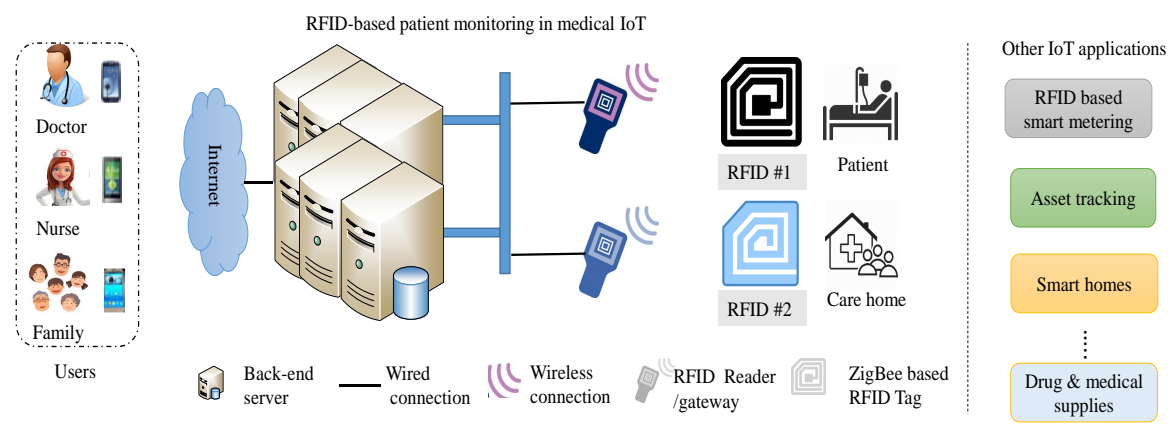

Fig. 2. RFID system architecture in IoT: a patient monitoring

Gope et al. presented a lightweight RFID authentication with privacy protection in smart city environment [7]. The scheme proposed in [7] not only defends the forward security, anonymity, and tag, but it also provides location privacy to the RFID-embedded devices. The scheme utilized hashing and XORing operations but it is still expensive for a RFID-tag as compared to state of the art protocols. Moreover, Shen et al. [8] pointed that the hashing and XORing based schemes are efficient but they cannot be implemented for many higher security attributes. Similar to the Gope et al.'s protocol, Liu et al. proposed an improvement on two-way authentication for RFID system [9]. The scheme is mainly focusing on security services but leaving out privacy issues. In addition, it is a common perception that symmetric key cryptographic and hashing primitives take less computational cost than the conventional public key cryptographic primitives. Vaudenay [10] has put an interesting observation on the issues of tracking attack in RFID system that says public key cryptography 
is needed in RFID authentication protocol to prevent the tracking attack. In addition, Vaudenay's work [10], [11] formalizes the notions of privacy of RFID system under the standard adversarial model. Subsequently, research progress on RFID authentication protocols $[12,13]$ has shown the feasibility of RFID authentication protocol implementation using ECC (elliptic curves cryptography [14]). Although, the ECC-based scheme would take more cost than the conventional symmetric key based scheme, a careful design of RFID authentication protocol [15] using ECC could resist tracking attack with a little more cost than symmetric key primitive but less cost in comparison to conventional public key primitive. Several works $[12,16-20,8]$ have been proposed in literature, claiming tracking resistant RFID authentication protocols, but later found them that they are either insecure against tracking attack [21-24] or required high computational and communicational costs, for instance, $[18,20,19,8]$. Furthermore, it is observed that many of these protocols use the concept of the Schnorr protocol [25] identification protocol. We note that the Schnorr protocol was designed for identification of the prover, not for tracking the prover. Because of this design criterion, many RFID authentication protocols which are based on the Schnorr protocol fail to defend the tracking attack.

Our contributions. To solve aforementioned issues, our contributions are as follows:

- We present an authentication protocol for RFID system that provides tag and reader mutual authentication and privacy preserving of tags. Elliptic curves are utilized for designing the protocol where underlying security assumption relies on Elliptic Curve Discrete Logarithm Problem and Decisional Diffie-Hellman assumption.

- The protocol is secure against narrow-strong adversary under standard assumption. We compare the protocol with some related work and show the usefulness of our protocol in emerging applications of RFID system.

- The proposed protocol is also extensible, that is, depending on RFID tags' usage in IoT applications the security goal of the proposed protocol can further be extended to achieve additional security services like session key establishment and data confidentiality.

Organization of the paper. The remainder of the paper is organized as follows. In section 2, we discuss the preliminaries, e.g., network model, RFID security and privacy issues followed by some computational assumptions on which the proposed protocol relies upon. In section 3, we present our protocol. We analyze security, and performance in section 4 and section 5, respectively. Finally, we conclude the paper in section 6 .

\section{Preliminaries}

\section{$2.1 \quad$ Network model}

With objects tagged with RFID and paired with an IoT application, consumers can improve their daily life and identify and track objects [3]. A typical ZigBee- 
enabled active RFID-based architecture in IoT applications is shown in Fig. 2 [26], where RFID-enabled patient is being monitored after patient hospitalization (e.g., after a cardiac operation). Under this architecture, a tag-enabled patient can talk to the reader-enabled bed and then, the reader can forward an alert message to the doctor. This is required because the tag-enabled patient should not wait for the reader's energy in getting activated, instead, the patient equipped with an active tag must trigger for an intended instruction to a nearby reader to reach out the doctor/nurse or family members to access healthcare services. Moreover, this system architecture can also expend to many other IoT applications, e.g., smart metering identification, asset tracking and management, smart homes, medical supplies and so on.

\subsection{Security and Privacy Properties of RFID System}

Typically, an RFID system aims to provide authentication and integrity as its security strength. In addition, RFID system should support tags' privacypreserving.

Security: Ensuring that fake tags are rejected when they communicate to a reader.

An RFID reader requires to identify whether the communicating tag is legitimate or fake. The reader should identify legitimate tags correctly and discard fake tag's request. As tag-reader communication goes through insecure radio waves, attacker can intercept any communications and then can manipulate towards his/her goal. Therefore, the communicating tag should send its identification information in such a way that should reach to the reader as a fresh request without being altered.

Privacy: Ensuring that privacy of the legitimate tags are protected while communicating with a reader.

RFID tags are typically attached to passports, consumer goods, library books, home appliances, healthcare equipments and other objects for identification and tracking purposes. Depending on various situations (e.g., misuse, stolen, or traitor tracing of tag-enabled items), the reader can trigger an appropriate message to seller/vendor/owner of the item in order to track the intended item. However, this cannot be generalized, as it compromises legitimate user's (e.g., tag-enabled item's owner) privacy. Therefore, privacy of tags is a crucial task to achieve in an RFID authentication protocol. On one hand, user who carries tag-enabled item should not be tracked which ensures it's privacy. On the other hand, with the help of tag-reader tracking feature, tag-enabled criminals or suspicious objects will get traced in a controlled manner that could save human lives and national assets (we note that this feature can be activated on a need-basis and with a genuine reason, such as law-enforcement purpose).

Efficiency: Typically a RFID tag is a resource constraint device, therefore, there should be a trade-off between the security, privacy and efficiency. In other words, the protocol must take communication and computation efficiency into consideration. 


\subsection{Computational Assumptions}

There are some standard (computationally) hard problems on which elliptic curve cryptographic primitives rely upon. Our proposed protocol relies on following two hard problems.

Elliptic Curve Discrete Logarithm Problem (ECDLP). The Elliptic Curve Discrete Logarithm Problem (ECDLP) is a standard assumption in which several ECC-based cryptographic algorithms can rely. The ECDLP is stated as: Given two elliptic curve points $P$ and $Q(=x P)$, finding the scalar $x$ is a hard problem.

Decisional Diffie-Hellman (DDH) assumption: Let $P$ be a generator of $E\left(F_{q}\right)$. Let $x, y, z \in_{R} Z_{q}, A=x P$, and $B=y P$. The $\mathrm{DDH}$ assumption is stated as: It is hard to distinguish two tuples $\langle A, B, x y P\rangle$ and $\langle A, B, z P\rangle$.

\section{Proposed Scheme}

Design goal and Assumptions. As shown in the previous section, many RFID authentication protocols do not support privacy preserving of tags. In practical applications of RFID system, preserving tag's (or tag-enabled object) privacy is an important requirement along with tag's authentication property. The proposed protocol aims to achieve two properties - (i) mutual authentication of tag and reader, and (i) tag's privacy preserving.

We assume that the back-end server is a trusted entity and the communication channel between the back-end server and reader is secure. We further assume that the back-end server personalizes all tags and readers with keying materials (e.g. public key and private key) before their deployment into the system. The public key of the tag and reader acts as the identification information, and the corresponding private key must be kept secret to the respective tag and reader. We also assume that tag has capabilities on computing basic ECC arithmetic and storing the required keying materials. The reader has more computing and storage capabilities than tag.

The protocol has two phases - Setup and Authentication. The Setup phase is a one-time computation that personalizes tags before their deployment into the field. The Setup phase is invoked when a new tag is added to the system or an exiting tag is removed/revoked from the system. The Authentication phase is a dynamic operation, where tag and reader interact to each other as and when required.

\subsection{Setup Phase}

The back-end server first selects a suitable elliptic curve group $E\left(F_{q}\right)$ over a finite field $F_{q}$, where $q$ is a prime number, sufficiently large enough to accommodate cryptographic keys. We assume that the back-end server initially personalizes $m$ number of tags and $n$ number of readers. The parameters $E\left(F_{q}\right), q$ and $P$ are made public, where $P \in E\left(F_{q}\right)$ is a generator of $E\left(F_{q}\right)$. 
- Setup Tag: For $i=1,2, \cdots, m$, the back-end server personalizes tag- $i$ with the private key $x_{i} \in Z_{q}^{*}$. It then computes tag- $i$ 's public key $X_{i}=x_{i} P$ and stores it in readers' database. The private key $x_{i}$ is known to the tag- $i$ only.

- Setup Reader: For $j=1,2, \cdots, n$, the back-end server personalizes reader$j$ with the private key $y_{j} \in Z_{q}^{*}$. It then computes reader-j's public key $Y_{j}=y_{j} P$ and stores it in tags' memory. The private key $y_{j}$ is known to the reader- $j$ only.

\subsection{Authentication Phase}

We note that the tag's public key $X$ acts its identity information which will link to its real identity $I D$ stored in reader's database during the Setup phase. The phase works as follows.

1. Tag $\rightarrow$ Reader: $\left\langle n_{s}\right.$, TID $>$

Tag chooses two random numbers $n_{s}, n_{t} \in Z_{q}^{*}$, and computes its transient identity

$$
T I D=n_{s} \cdot n_{t} \cdot X
$$

Tag then sends $<n_{s}, T I D>$ to the reader.

2. Reader $\rightarrow$ Tag: $<R I D$, Auth $_{r}>$

Upon receiving tag's request, the reader chooses a random number $n_{r} \in Z_{q}^{*}$ and computes

$$
\begin{aligned}
& R I D=n_{s} \cdot n_{r} \cdot Y \\
& C=y \cdot n_{r} \cdot T I D \\
& \text { Auth }_{r}=\operatorname{PRF}\left(\bar{Y}, \bar{C}, \bar{X}, n_{s}\right)
\end{aligned}
$$

Here, $R I D$ is the reader's transient identity, $C$ is a challenge, and $A u t h_{r}$ is the authentication code for reader's authenticity confirmation. The $\bar{\lambda}$ indicates the $\mathrm{x}$-coordinate of the point $\lambda$ for $\lambda \in\{X, Y, C\}$. The $(P R F()$ is a pseudo-random function used for authentication code generation. We consider Advanced Encryption Standard (AES)-based message authentication code such as cipher block chaining (CBC) because of its efficiency in comparison to conventional hash function based message authentication code.

The reader sends $<R I D$, Auth $_{r}>$ to the tag.

3. Tag $\rightarrow$ Reader: $\left\langle\right.$ Auth $\left._{t}\right\rangle$

After receiving $<R I D$, Auth $_{r}>$ from the reader, tag computes $C^{\prime}=x$. $n_{t} \cdot R I D$ and checks whether $A u t h_{r}=\operatorname{PRF}\left(\bar{Y}, \bar{C}^{\prime}, \bar{X}, n_{s}\right)$. If it holds, then reader's authentication is confirmed. Now the tag computes

$A u t h_{t}=\operatorname{PRF}\left(\bar{X}, \bar{C}^{\prime}, R \bar{I} D, n_{s}\right)$ and sends $<A u t h_{t}>$ to the reader as the response to reader's challenge.

4. After receiving $\left\langle A u t h_{t}\right\rangle$, the reader validates $\left\langle A u t h_{t}\right\rangle$ by checking whether $A u t h_{t}=\operatorname{PRF}\left(\bar{X}, \bar{C}, R \bar{I} D, n_{s}\right)$

If it holds, then tag's authentication is confirmed. The flow of scheme is shown in Fig. 3

We note that the authentication code $A u t h_{r}$ is computed by the transient secret $C$ generated by the reader (and $A u t h_{t}$ by the transient secret $C^{\prime}$ of the tag). 


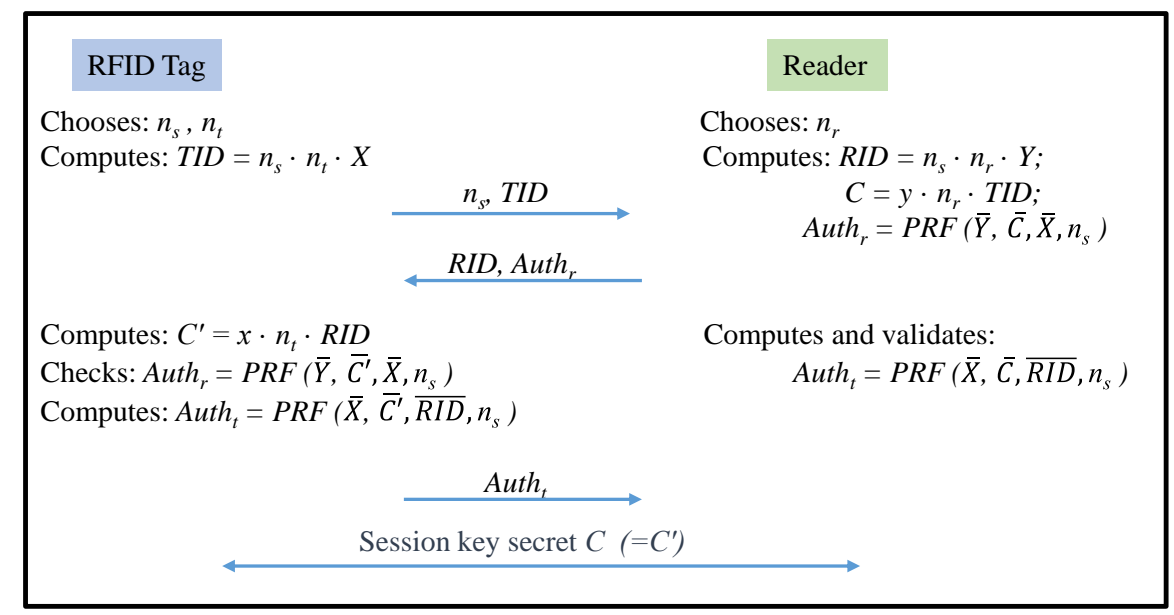

Fig. 3. Flow of the proposed Scheme - Authentication phase

These two transient secrets are same, as checked by the following correctness:

$$
\begin{aligned}
C & =y \cdot n_{r} \cdot T I D \\
& =y \cdot n_{r} \cdot n_{s} \cdot n_{t} \cdot X \\
& =y \cdot n_{r} \cdot n_{s} \cdot n_{t} \cdot x \cdot P \\
& =x \cdot n_{t} \cdot n_{s} \cdot n_{r} \cdot y \cdot P \\
& =x \cdot n_{t} \cdot n_{s} \cdot n_{r} \cdot Y \\
& =x \cdot n_{t} \cdot R I D \\
& =C^{\prime}
\end{aligned}
$$

We note that in Step 2, the reader uses tag's public key $X$ to compute $A u t h_{r}$. Therefore, the reader should have information of the tag's public key $X$ in order to execute Step 2. The information of tag's $X$ can be communicated through a separate parameter $\alpha=n_{a} Y \oplus X \oplus Y$ along with $A=n_{a} P$, where $n_{a}$ is a random integer in $Z_{q}^{*}, P$ is a generator of $E\left(F_{q}\right)$. The reader can retrieve $X$ by computing $\alpha \oplus Y \oplus y A$ and then checks with its database for any entry of $X$. If so, the reader picks that $X$ and computes $A u t h_{r}$, else, the reader rejects the tag's request.

\section{Security Analysis}

In this section, we analyse the security of proposed scheme.

An RFID system can have multiple readers. However, for the sake of simplicity, we assume that the system consists of one reader and a set of tags $\mathcal{T}=\left\{T_{1}, T_{2}, \cdots, T_{m}\right\}$. The back-end server is a trusted entity, connected to the reader via a secure channel. Although the back-end server is connected to the reader securely for identifying the communicating tag, we simplify it by con- 
sidering the reader and the back-end server as a single entity. We assume that the adversary can compromise one or more tags, but he cannot compromise the reader. Initially, $\mathcal{T}$ is empty, and then new tags are added to the system as and when required. The reader maintains a database of tuples $\left\langle I D_{i}, X_{i}\right\rangle$ for every $\operatorname{tag} T_{i} \in \mathcal{T}$, where the tag's identity $I D_{i}$ for $i=1,2, \cdots, m$ is logically linked to its public key $X_{i}$. We note that the public key $X_{i}$ of the tag- $i$ provides tag's authenticity to the reader, therefore, the public key of the tag needs to be protected as long as tag's privacy is concerned. Therefore, the public key of the tag is not publicly available, instead, the public keys of the communicating tags are known to reader only, and the public key of the reader is known to tags only. Every tag $T_{i}$ maintains an internal state $S_{i}$ in the proposed scheme.

Theorem 1. The proposed protocol preserves narrow-strong privacy under the DDH assumption.

Proof. Suppose that an adversary $\mathcal{A}$ can break the narrow-strong privacy of the protocol. Then, $\mathcal{A}$ is able to distinguish a tag from different instances of the protocol run. Now, in order to break the privacy of the protocol, $\mathcal{A}$ gathers sufficient information through the Learning phase and then faces to Challenge phase as explained below.

Learning phase: The adversary $\mathcal{A}$ gathers $\left(R I D_{r_{i}}, A u t h_{r_{i}}\right)$ for $i=1,2, \cdots, q_{r}$, and $\left(n_{s_{i}}, T I D_{t_{i}}\right.$, Auth $\left._{t_{i}}\right)$ for $i=1,2, \cdots, q_{t}$.

We assume that $\mathcal{A}$ can corrupt any $m-2$ tags (except 2 tags). He then obtains the non-volatile memory status $\mathcal{S}$ of $m-2$ corrupt tags. For simplicity, we assume that tags $T_{3}, T_{4}, \cdots, T_{m}$ are corrupt tags, and $T_{1}$ and $T_{2}$ are not corrupt. By compromising $T_{3}, T_{4}, \cdots, T_{m}$, the adversary $\mathcal{A}$ has knowledge of secrets $x_{3}, x_{4}, \cdots, x_{m}$, and the public key of the reader $Y$.

Challenge phase: The challenger $\mathcal{C}$ picks $b \in_{R}\{0,1\}$ and submits following to $\mathcal{A}$ :

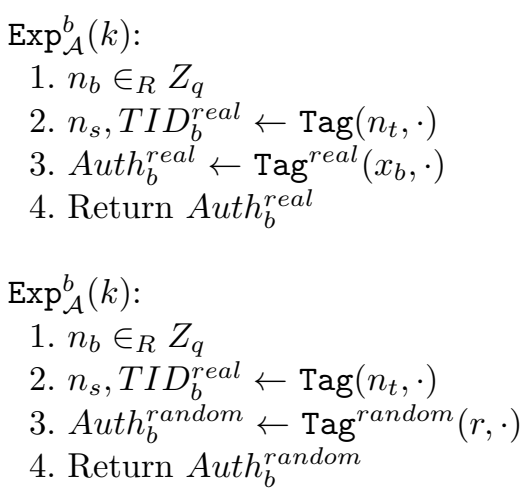

$\mathcal{A}$ 's task is to guess whether $A u t h_{b}^{\text {random }} \in\left\{T_{1}, T_{2}\right\}$. It is computationally infeasible for $\mathcal{A}$ to find any clue of $n_{t}$ from $T I D_{b}^{\text {real }}$, as $T_{b}^{\text {real }}=n_{s} n_{t} X$, and the problem is to solve the ECDLP, which is a hard problem.

Now, $\mathcal{A}$ can use his gathered information from the Learning phase to check whether $A u t h_{b}^{\text {real }}$ and $A u t h_{b}^{\text {random }}$ match to any records $<R I D_{r_{i}}, A u t h_{r_{i}}>$ 
and $<T I D_{t_{j}}$, Auth $_{t_{j}}>$ for tags $T_{3}, T_{4}, \cdots, T_{m}$, where $i=1,2, \cdots, q_{r}$ and $j=1,2, \cdots, q_{t}$. However, none of these records in $\mathcal{A}$ 's database can find any mapping to $A u t h_{b}^{\text {real }}$ or $A u t h_{b}^{\text {random }}$. Therefore, $\mathcal{A}$ 's guess to check if $A$ uth ${ }_{b}^{\text {random }} \in$ $\left\{T_{1}, T_{2}\right\}$ would give him a success probability $\frac{1}{2}$.

Therefore, given $<P, T I D_{b}^{\text {real }}$, Auth $h_{b}^{\text {real }}>$ and $<P, T I D_{b}^{\text {real }}$, Auth $h_{b}^{\text {random }}>$, the adversary $\mathcal{A}$ cannot say whether $A$ uth ${ }_{b}^{\text {random }} \in\left\{T_{1}, T_{2}\right\}$ with probability significantly greater than $\frac{1}{2}$. The reason is that the complexity of solving this problem reduces to the DDH problem, which is a hard problem. As a result, $\mathcal{A}$ can not distinguish a tag from different instances of the protocol run.

Hence, the advantage $\operatorname{Adv}_{\mathcal{A}}(k)$ of the privacy experiment that $\mathcal{A}$ can have in our protocol is probability $\frac{1}{2}+2^{-k}$, which is approximately same probability of a random guess, that is, $\frac{1}{2}$.

Theorem 2. The proposed protocol is safe against replay attack.

Proof. Assumed that an attacker captures $\left\langle n_{S}, T I D\right\rangle,<R I D$, Tut $\left.h_{r}\right\rangle$ and $<$ $A u t h_{t}>$ message via eavesdropping. Later, he/she tries to replay $\left\langle n_{S}, T I D\right\rangle$ to the reader. However, due to the fact of the nonce, the message $\left\langle n_{S}, T I D\right\rangle$ will not be verified at the reader. Thus, the replayed message will be detected. Likewise, the other messages, e.g., $\left\langle R I D\right.$, Tuth $\left._{r}\right\rangle$ and $\left\langle A u t h_{t}\right\rangle$ are also be secured against the replay attack.

Theorem 2. The proposed scheme is safe against impersonation attack

Proof. An attacker may try to impersonate the tag to the reader. In this attempt, an attacker sends $\left\langle n_{a t t}, T I D\right\rangle$ to reader. However, this message cannot verified at the reader as $T I D$ is being computed via two random numbers $\left(n_{s} \cdot n_{t} . X\right)$, therefore TID cannot be verified with $n_{a t t}$ at the reader. Thus, the proposed scheme is safe against the impersonation attack. Likewise, the attacker cannot impersonate the reader.

Theorem 3. Mutual authentication and secure key establishment

Proof. In the proposed scheme, both the entities (i.e., tag and reader) perform mutual authentication and establish the secure key. As shown in Fig. 3, the tag verifies the sub-message $A u t h_{r}\left(=\operatorname{PRF}\left(\bar{Y}, \bar{C}^{\prime}, \bar{X}, n_{s}\right)\right)$ and assures that < $R I D, A u t h_{r}>$ is generated by the legitimate reader. Similarly, the reader verifies the sub-message $A u t h_{t}$ and assures that $<A u t h_{t}\left(=\operatorname{PRF}\left(\bar{X}, \bar{C}, R \bar{I} D, n_{s}\right)\right)>$ is generated by the legitimate tag. Upon successful mutual authentication both the entities can establish a common secret $C\left(=C^{\prime}\right)$. Using this common secret and other parameters (e.g., random numbers, public key) they can derive secret keys (e.g., encryption key, update key).

Theorem 4. Provides secure message transmission

Proof As it can be noticed in Fig. 3, both tag and reader can compute the session key by which they can securely transmit message in a confidential manner and achieve message privacy.

Theorem 5. The proposed scheme achieves forward secrecy and privacy 
Proof. By saying forward privacy we mean if the adversary gets hold of the private key $x$ of the tag then he can not relate to any previously messages intercepted from tag-reader communication where the same tag might have been participated. We note that the ephemeral secrets $n_{t}$ and $n_{s}$ are deleted from the local state of the tag and the reader once the session is over or expired. Furthermore, the adversary can not guess $n_{t}$ from TID (resp. $n_{r}$ from RID), as it relies on ECDLP, which is an intractable problem. Even if the adversary knows the private key $x$ of the tag and private key $y$ of the reader, he can not compute $C^{\prime}$ (resp. C) without knowing the corresponding $n_{t}$ (resp. $\left.n_{r}\right)$. As we can see in the authentication protocol, both the tag and the reader agree on a common secret $C$ or $C^{\prime}$ pertaining to the session specific ephemeral secrets $n_{t}$ and $n_{r}$. These numbers are kept secret to their local states and deleted from the local states once the session gets expired. Without knowing session specific $C$ (resp. $C^{\prime}$ ) or $n_{r}$ (resp. $\left.n_{t}\right)$ pertaining to session, he can not relate to any previously intercepted messages (e.g., Auth ${ }_{r}$, Auth $_{t}$ ) in the protocol run where the same tag might have been participated. Therefore, the protocol provides.

Theorem 6. The proposed scheme achieves anonymity and untraceability

Proof. Assumed that an attacker may utilize a powerful resource to intercept the messages between the tag and reader to detect the identities of communicating entities. However, in the proposed scheme the messages are being generated using the random numbers, e.g., $n_{t}, n_{s}, n_{r}$. As, the random numbers provide enough randomness for each session, the attacker would not able to obtain and trace the real identities of communicating entities. Thus, the proposed scheme achieves anonymity and untraceability.

Finally, Table 1 summarizes the security comparison of the proposed scheme and other state-of-the-art schemes. It can be seen from Table 1, the proposed scheme provides more security and privacy services than the others.

Table 1. Comparisons of security services

\begin{tabular}{|l|l|l|l|l|l|l|}
\hline & {$[8]$} & {$[17]$} & {$[18]$} & {$[19]$} & {$[20]$} & Ours \\
\hline Mutual Authentication & $\checkmark$ & $\checkmark$ & $\checkmark$ & $\checkmark$ & $\checkmark$ & $\checkmark$ \\
\hline Session key establishment & ND & ND & ND & ND & - & $\checkmark$ \\
\hline Confidentiality & $\checkmark$ & ND & $\checkmark$ & $\checkmark$ & $\checkmark$ & $\checkmark$ \\
\hline Forward security & $\checkmark$ & ND & $\checkmark$ & $\checkmark$ & ND & $\checkmark$ \\
\hline Anonymity & $\checkmark$ & $\checkmark$ & $\checkmark$ & $\checkmark$ & $\checkmark$ & $\checkmark$ \\
\hline Untraceability & $\checkmark$ & ND & ND & ND & ND & $\checkmark$ \\
\hline
\end{tabular}

ND: no discussion 


\section{Implementation and Performance Analysis}

This section evaluates the proposed scheme by simulating the ECC operations on the Texas Instruments designed MSP430 microcontrollers integrated with 16-bit RISC processor (i.e., $8 \mathrm{MHz}$ frequency). The MSP430 microcontroller can be operated at low voltages, for instance, 1.8 to $3.3 \mathrm{~V}$ [27]. From the security perspective, a MSP430 microcontroller is integrated with the AES hardware accelerator that can support 128-bit encryption. Moreover, this family (i.e., MSP430) can be used for low-power IoT applications (such as, medical, environment monitoring, and so on). Our experimental setup is shown in Fig. 4. Consider a patient monitoring scenario (please refer to Fig. 2), where a MSP430 tag is attached to a patient. The tag transmits patient's information via the reader and/or gateway to the backend (e.g., Intel $2.59 \mathrm{GHz}$, and $16 \mathrm{~GB}$ of RAM), i.e., a laptop in our experiment setting. To support ECC operations, we used TinyECC [28] software library and ECC optimizations [29]. In addition, as mentioned in the protocol design, for the sake of implementation purpose, the protocol uses a $\operatorname{PRF}()$ for message authentication code (MAC) generation that can be used a lightweight MAC via (AES) cipher block chaining (AES-CBC).

Note that as the computational power of a tag is limited, we evaluated the security prices of the proposed protocol at tag only. The implementation uses following message sizes, for instance, a random number $=32$-bit, AES-MAC (as PRF) $=64$-bit, and we selected to use secp160r1 defined over a 160-bit prime field. Therefore, the length of protocol messages (refer to Fig. 3), i.e., $\left\langle n_{s}, T I D\right\rangle$, $<R I D, A u t h_{r}>$, and $<A u t h_{t}>$ are 192-bit, 224-bit, and 64-bit long, respectively. The measurement results of our implementations, for instance, a point multiplication and a PRF (i.e., AES-MAC) are $2876 \mathrm{~ms}$, and $7.8 \mathrm{~ms}$, respectively, at the tag side.
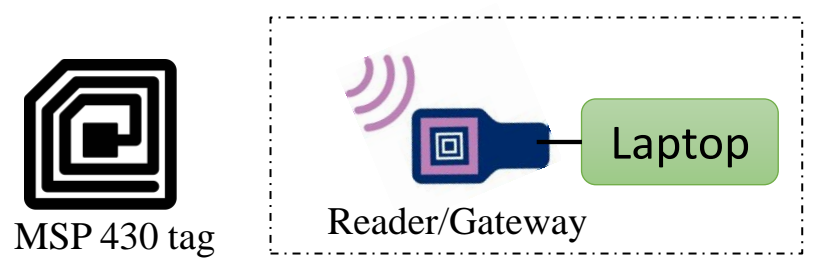

Fig. 4. Experimental Setup

Now, we illustrate computational and communication costs of the proposed protocol and compare it with those schemes that utilize the ECC operations, for instance, Shen et al. [8], Lee et al. [17], He et al. [18], Jin et al. [19], Chien [20], and Bringer et al. [21]. The computation cost comparison is given in Table 
Table 2. Comparisons of computational cost

\begin{tabular}{|l|l|l|l|}
\hline & At Tag & At Reader & Total \\
\hline Shen et al. $[8]$ & $3 \mathrm{M}+2 \mathrm{~A}+3 \mathrm{H}$ & $3 \mathrm{M}+1 \mathrm{~A}+3 \mathrm{H}$ & $6 \mathrm{M}+3 \mathrm{~A}+6 \mathrm{H}$ \\
\hline Lee et al. $[17]$ & $4 \mathrm{M}$ & $3 \mathrm{M}+1 \mathrm{~A}$ & $7 \mathrm{M}+1 \mathrm{~A}$ \\
\hline He et al. $[18]$ & $3 \mathrm{M}$ & $3 \mathrm{M}$ & $6 \mathrm{M}$ \\
\hline Jin et al. $[19]$ & $4 \mathrm{M}+2 \mathrm{~A}+2 \mathrm{H}+1 \mathrm{E}$ & $4 \mathrm{M}+2 \mathrm{~A}+2 \mathrm{H}+1 \mathrm{E}$ & $8 \mathrm{M}+4 \mathrm{~A}+4 \mathrm{H}+1 \mathrm{E}+1 \mathrm{D}$ \\
\hline Chien $[20]$ & $3 \mathrm{M}+1 \mathrm{~A}+4 \mathrm{H}$ & $3 \mathrm{M}+1 \mathrm{~A}+4 \mathrm{H}$ & $6 \mathrm{M}+1 \mathrm{~A}+8 \mathrm{H}$ \\
\hline Bringer et al. $[21]$ & $2 \mathrm{M}+1 \mathrm{~A}$ & $3 \mathrm{M}+2 \mathrm{~A}$ & $5 \mathrm{M}+3 \mathrm{~A}$ \\
\hline Our Scheme & $2 \mathrm{M}+2 \mathrm{PF}$ & $2 \mathrm{M}+2 \mathrm{PF}$ & $4 \mathrm{M}+4 \mathrm{PF}$ \\
\hline
\end{tabular}

M: the time for executing point multiplication operation, A: the time for executing addition operation, $\mathrm{PF}$ : the time for executing $\mathrm{PRF}()$ computation, $\mathrm{H}$ : the time for executing hash operation, E: the time for executing Encryption, D: the time for executing decryption

2 and it is easy to see that the schemes proposed in [8], [17], [19], [20], and [21] required $3 \mathrm{M}+2 \mathrm{~A}+3 \mathrm{H}, 4 \mathrm{M}, 3 \mathrm{M}, 4 \mathrm{M}+2 \mathrm{~A}+2 \mathrm{H}+1 \mathrm{E}, 3 \mathrm{M}+1 \mathrm{~A}+4 \mathrm{H}$, and $2 \mathrm{M}+1 \mathrm{~A}$, respectively, at the tag side. Whereas, the proposed protocol requires $2 \mathrm{EC}$ point multiplications and $2 \mathrm{PRF}()$ computations on the patient's tag, which reduces the computational burden on the tag as compared to other schemes. Moreover, it can also be noticed that the proposed scheme is not only efficient at the tag but it also required low computation cost in total ( i.e., $4 \mathrm{M}+4 \mathrm{PF}$ ) in contrast to other schemes, as shown in Table 2. Hence, the proposed scheme can achieve computational efficiency at (low power) RFID IoT applications. It is widely known that to transmit a bit required more energy than computing a bit. As many of RFID enabled objects are typically power-hungry, communicational cost (i.e., number of bits to be transmitted) is one of the main concerns IoT applications. However, the proposed scheme required three messages exchanges between the tag and the reader, as shown in Fig. 3. In the proposed scheme, a tag sends two messages (i.e., $\left\langle n_{s}, T I D>\right.$ and $\left.<A u t h_{t}\right\rangle$ ) and receives one message (i.e., $<R I D$, Auth $_{r}>$ ). Table 3 summarizes the number of bits transmitted and received at the tag for the proposed scheme and [8], [18], [19], [20]. As seen from Table 3, the proposed scheme required only 496-bit communication overhead to execute the whole protocol, whereas the schemes proposed in $[8],[18],[19],[20]$ required more communicational overhead. In addition, Fig. 5 depicts the number of message exchanges of the proposed scheme and [8], [18], [19], [20]. Shen et al.'s scheme requires two message exchanges, however, it needs to communicate a high number of bits (i.e., 1400), as shown in Table 3. Our scheme requires three message exchanges as in [18], [19], [20] but it needs to communicate only 496-bit to complete the protocol, which incurs low communication burden than the other schemes. 
Table 3. Comparisons of computational cost at the tag

\begin{tabular}{|l|l|l|l|}
\hline & Send (in bits) & Receive (in bits) & Total \\
\hline Shen et al. [8] & 640 & 800 & 1440 \\
\hline He et al. [18] & 640 & 640 & 1280 \\
\hline Jin et al. [19] & - & - & 1120 \\
\hline Chien [20] & 480 & 320 & 800 \\
\hline Our Scheme & 256 & 240 & 496 \\
\hline
\end{tabular}

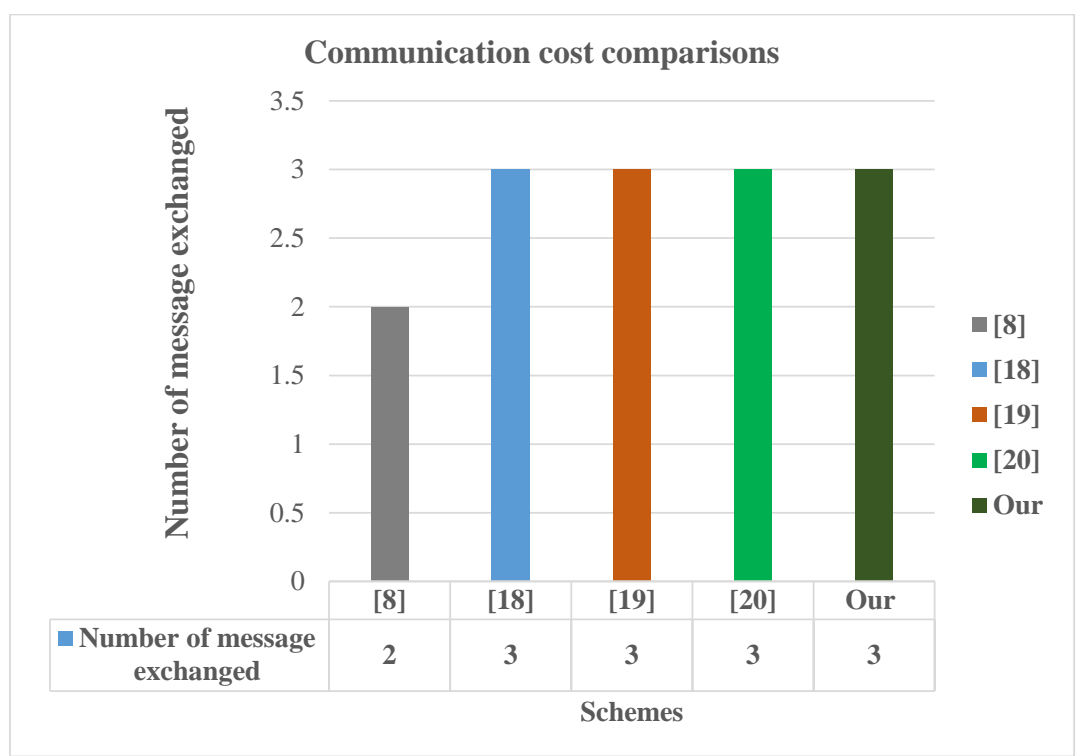

Fig. 5. Communication cost comparisons. 


\section{Conclusion}

In a recent market report, the global RFID technology is projected to cross $\$ 40.5$ billion by 2025 . As per the report, RFIDs are increasing applications ranging from consumer electronics (e.g., milk pouch) to border security (e.g., passport). Privacy issue of tag-enabled IoT objects has become an important concern in RFID system. We proposed an RFID authentication protocol using elliptic curves arithmetic for narrow-strong privacy-preserving of tags. The protocol also provides additional security services like key establishment and data confidentiality, which may be needed for future Internet applications of RFID system. Moreover, the proposed scheme can be expended to many other IoT applications, e.g., smart metering identification, asset tracking and management, smart homes, medical supplies and so on, where efficiency, security and privacy are highly required.

\section{References}

1. S. Naskar, P. Basu, and A. K. Sen. A literature review of the emerging field of IOT using RFID and its applications in supply chain management. In the Internet of Things in the Modern Business Environment Advances in E-Business Research, pp. 1-27, 2017.

2. J. D. Irawan, E. Adriantantri, and A. Farid. RFID and IOT for attendance monitoring system. In MATEC Web of Conferences, vol. 164. EDP Sciences, 2018.

3. P. Porambage, M. Ylianttila, C. Schmitt, P. Kumar, A. Gurtov, and A. V. Vasilakos. The quest for privacy in the internet of things. In IEEE Cloud Computing, vol. 3, no. 2, pp. 36-45, 2016.

4. H. Mahdin, H. Kamaludin, R. R. Saedudin, A. H. Omar, S. Kasim, and J. Jailani. The application of RFID system in water level monitoring. In International Journal on Advanced Science, Engineering and Information Technology, vol. 7, no. 4-2, pp. 1522-1527, 2017.

5. K. Fan, W. Jiang, H. Li, and Y. Yang. Lightweight RFID protocol for medical privacy protection in IOT. In IEEE Transactions on Industrial Informatics, vol. 14, no. 4, pp. 1656-1665, 2018.

6. F. Rahman, M. Z. A. Bhuiyan, and S. I. Ahamed. A privacy preserving framework for RFID based health care systems. In Future Generation Computer Systems, vol. 72, pp. 339-352, 2017.

7. P. Gope, R. Amin, S. H. Islam, N. Kumar, and V. K. Bhalla. Lightweight and privacy-preserving RFID authentication scheme for distributed IOT infrastructure with secure localization services for smart city environment. In Future Generation Computer Systems, 2017.

8. H. Shen, J. Shen, M. K. Khan, and J.-H. Lee. Efficient RFID authentication using elliptic curve cryptography for the internet of things. In Wireless Personal Communications, vol. 96, no. 4, pp. 5253-5266, 2017.

9. B. Liu, B. Yang, and X. Su. An improved two-way security authentication protocol for RFID system. In Information, vol. 9, no. 4, 2018.

10. S. Vaudenay. On privacy models for RFID. In International Conference on the Theory and Application of Cryptology and Information Security, pp. 68-87. Springer, 2007. 
11. J. Hermans, R. Peeters, and B. Preneel. Proper RFID privacy: model and protocols. In IEEE Transactions on Mobile Computing, vol. 13, no. 12, pp. 2888-2902, 2014.

12. Y. K. Lee, K. Sakiyama, L. Batina, and I. Verbauwhede. Elliptic-curve based security processor for RFID. In IEEE Transactions on Computers, vol. 57, no. 11, pp. 1514-1527, 2008.

13. D. Hein, J. Wolkerstorfer, and N. Felber. ECC is ready for RFID-a proof in silicon. In International Workshop on Selected Areas in Cryptography, pp. 401-413. Springer, 2008.

14. D. Hankerson, A. J. Menezes, and S. Vanstone. Guide to elliptic curve cryptography. In Springer Science \& Business Media, 2006.

15. M. L. Das. Strong security and privacy of RFID system for internet of things infrastructure. In International Conference on Security, Privacy, and Applied Cryptography Engineering, pp. 56-69. Springer, 2013.

16. Y. K. Lee, L. Batina, and I. Verbauwhede. Untraceable RFID authentication protocols: Revision of EC-RAC. In IEEE International Conference on RFID, pp. 178$185,2009$.

17. Y. K. Lee, L. Batina, D. Singelee, and I. Verbauwhede. Low-cost untraceable authentication protocols for RFID. In Proceedings of the third ACM conference on Wireless network security, pp. 55-64. 2010.

18. D. He, N. Kumar, N. Chilamkurti, and J.-H. Lee. Lightweight ECC based RFID authentication integrated with an ID verifier transfer protocol. In Journal of Medical Systems, vol. 38, no. 10, p. 116, 2014.

19. C. Jin, C. Xu, X. Zhang, and J. Zhao. A secure RFID mutual authentication protocol for healthcare environments using elliptic curve cryptography. In Journal of Medical Systems, vol. 39, no. 3, p. 24, 2015.

20. H.-Y. Chien. Elliptic curve cryptography-based RFID authentication resisting active tracking. In Wireless Personal Communications, vol. 94, no. 4, pp. 2925-2936, 2017.

21. J. Bringer, H. Chabanne, and T. Icart. Cryptanalysis of EC-RAC, a RFID identification protocol. In International Conference on Cryptology and Network Security, pp. 149-161, Springer, 2008.

22. T. Van Deursen and S. Radomirovic. Attacks on RFID protocols. In IACR Cryptology ePrint Archive, vol. 2008, no. 310, pp. 1-56, 2008.

23. T. Van Deursen and S. Radomirovic. EC-RAC: enriching a capacious RFID attack collection. In International Workshop on Radio Frequency Identification: Security and Privacy Issues, pp. 75-90. Springer, 2010.

24. J. Pokala, M. C. Reddy, S. Bapana, C. S. Vorugunti et al. A secure RFID protocol for telecare medicine information systems using ECC. In International Conference on Wireless Communications, Signal Processing and Networking, pp. 2295-2300, 2016.

25. C.-P. Schnorr. Efficient identification and signatures for smart cards. In Conference on the Theory and Application of Cryptology, pp. 239-252. Springer, 1989.

26. T. Adame, A. Bel, A. Carreras, J. Melia-Segui, M. Oliver, and R. Pous. Cuidats: An RFID-WSN hybrid monitoring system for smart health care environments. In Future Generation Computer Systems, vol. 78, pp. 602-615, 2018.

27. G. Hinterwalder, A. Moradi, M. Hutter, P. Schwabe, and C. Paar. Fullsize highsecurity ECC implementation on msp430 microcontrollers. In International Conference on Cryptology and Information Security in Latin America, pp. 31-47, Springer, 2014. 
28. A. Liu and P. Ning. Tinyecc: A configurable library for elliptic curve cryptography in wireless sensor networks. In Proceedings of the 7 th international conference on Information processing in sensor networks, pp. 245-256, 2008.

29. L. Marin, A. Jara, and A. S. Gomez. Shifting primes: Optimizing elliptic curve cryptography for 16-bit devices without hardware multiplier. In Mathematical and Computer Modelling, vol. 58, no. 5-6, pp. 1155-1174, 2013. 